\title{
Chapter C3
}

\section{AXONS AND NEURONS IN CORONAVIRUS- INDUCED DEMYELINATION}

\author{
Ajai A. Dandekar and Stanley Perlman \\ Departments of Pediatrics and Microbiology and Interdisciplinary Program in Immunology, \\ Univerisity of Iowa, Iowa City, Iowa, USA
}

\begin{abstract}
Infection of mice with the coronavirus mouse hepatitis virus induces primary demyelination in susceptible strains of rodents. Although demyelination is the primary pathological process detected in the central nervous system of infected mice, axonal dysfunction and damage also occur concomitantly with demyelination. This process is $\mathrm{T}$ cell mediated, with either CD4 or CD8 T cells sufficient for MHV-induced axonal damage. A striking feature is that axonal damage occurs early in the disease process, at nearly the same time as demyelination is first observed. Axonal damage in MHV-infected mice has many similarities with the parallel process in humans with multiple sclerosis.
\end{abstract}

Key words: Coronavirus, demyelination, axonal damage, $\mathrm{T}$ cells

\section{INTRODUCTION}

The human disease multiple sclerosis (MS) is characterized by focal demyelinating lesions throughout the white matter of the CNS $(1,2)$. In multiple sclerosis, immune-mediated damage to oligodendrocytes and/or the myelin sheaths accounts for this pathology. The process of demyelination results in electrical conduction deficits and other alterations in axonal physiology, and previously had been assumed to account for the clinical signs and symptoms of MS.

As described elsewhere in this volume, several older studies identified axonal damage as part of the disease process in MS (3). A series of recent reports confirmed that permanent damage to neurons and their axons also occurs within these demyelinating lesions (4-6). In one study (7), N-acetyl 
aspartate, a neurotransmitter, was diminished throughout the CNS in patients with progressive MS, while those patients with relapsing-remitting MS exhibited reduced levels of $\mathrm{N}$-acetyl aspartate only in areas of demyelination. This reduction in $\mathrm{N}$-acetyl aspartate correlated with axonal loss detected by either magnetic resonance imaging or electron microscopy of involved tissue.

Other studies of patients with MS have examined the characteristics of axonal damage, using immunohistochemistry with antibodies to nonphosphoneurofilament $\mathrm{H}$ or amyloid precursor protein to visualize damaged axons $(5,6)$. The preponderance of axonal damage occurred in demyelinating lesions, with the remainder of damage found adjacent to these lesions.

Axonal damage in MS may account for a significant amount of the clinical signs and symptoms seen in the progressive phase of disease. Progressive MS is poorly correlated with the size or number of demyelinating lesion seen by MRI, suggesting that axonal pathology, not demyelination, may be the primary cause of the irreversible deficits that are observed (8). One interpretation is that demyelination and remyelination may explain the relapsing-remitting phase, while progressive disease results from irreversible axonal pathology (9).

Axonal damage has been reported in several animal models of MS, including in rodents with experimental autoimmune encephalomyelitis (EAE) and in mice with demyelination induced by Theiler's encephalomyelitis virus or mouse hepatitis virus, strain JHM (MHV) (1013). Although it had long been believed that MHV-associated demyelination occurs primarily as a result of virus-induced destruction of oligodendrocytes, accumulating data from multiple studies indicate that CD4 and CD8 T cells are essential in the pathological process $(14,15)$ (see also chapter $\mathrm{C} 4$ of this volume). In this review, we summarize data showing that this $\mathrm{T}$ cellmediated demyelination occurs concomitantly with axonal damage in MHVinfected mice.

\section{TEMPOROSPATIAL PROFILE OF AXONAL DAMAGE IN MHV INFECTION}

As described elsewhere in this volume, several models of MHV-induced demyelination are studied in different laboratories. In one model, splenocytes are transferred from MHV immune mice to syngeneic immunodeficient mice [mice with severe combined immunodeficiency or with genetic disruption of recombination activation gene $1\left(\mathrm{RAGl}^{-1-}\right)$ ] infected with the neuroattenuated variant of MHV, 2.2-V-1 (16). Both SCID 
and $\mathrm{RAG1}^{-1-}$ mice lack B and T lymphocytes (17) and as such are unable to mount an adaptive immune response to MHV. While immunocompetent C57B1/6 (B6) mice develop demyelination ten to twelve days after intracranial inoculation with $\mathrm{MHV}$, their $\mathrm{RAG}^{-/}$or SCID counterparts develop, instead, a fatal acute encephalitis at 14-18 days post infection (p.i.). Clinical and histological evidence of demyelination, with accompanying macrophage/microglia infiltration, can be detected within seven to ten days of transfer.

Most recent work has been performed using MHV-infected RAG $1^{-/}$mice as recipients. In this adoptive transfer model, either CD4 or CD8 T cells can mediate demyelination and disease. $\mathrm{T}$ cells are necessary for the demyelinating process since transfer of splenocytes depleted of CD4 and $\mathrm{CD} 8 \mathrm{~T}$ cells results in no demyelination. $\mathrm{RAG}^{\%}{ }^{\%}$ recipients of $\mathrm{CD} 4 \mathrm{~T}$ cellenriched splenocytes (CD8 T cell-depleted) or CD8 $\mathrm{T}$ cell-enriched splenocytes (CD4 $\mathrm{T}$ cell-depleted) also develop demyelination, although with different kinetics than recipients of undepleted splenocytes. CD4 T cell enrichment results in a rapid course of disease, with mortality by day 7 p.i., while CD8 $\mathrm{T}$ cell enrichment results in a protracted course of disease (as compared to wild type), with mortality at day 14-16 p.i. or later. Strikingly, up to $50 \%$ demyelination is observed in recipients of CD8 T cell-enriched populations by 15 days p.i. This model system was used to determine the kinetics of axonal damage and relationship to demyelination in MHVinfected mice.

\subsection{The relationship between demyelination and axonal damage in MHV-induced disease}

Spinal cords were harvested from MHV-infected RAG1-/- mice at 7 days after adoptive transfer (10 days p.i.) of MHV immune splenocytes. We examined zinc formalin-fixed, paraffin-embedded sections for demyelination and axonal damage. Areas of myelin damage were determined using the chemical stain luxol fast blue (LFB) with quantification as previously described (18). We further assessed the distribution of macrophages/microglia using $\mathrm{mAb}$ to the macrophage-specific protein $\mathrm{F} 4 / 80$ (Serotec, Oxford, England) and viral antigen (using the MHV nucleocapsidspecific mAb 5B11.2, provided by Dr. M. Buchmeier, The Scripps Research Institute, La Jolla, CA) by immunohistochemistry. Additionally we stained for axonal damage using an antibody specific for nonphosphoneurofilament $\mathrm{H}$ (mAb SMI-32 (Sternberger Monoclonals, Lutherville, MD). This protein is largely found in damaged axons, but is also expressed in the cell body and proximal processes of a fraction of unaffected neurons. 
In areas of demyelination, there was abundant staining with SMI-32, indicating axonal damage. Examination of the staining pattern revealed continuous axonal staining, suggestive of intact, demyelinated axons, discontinuous staining patterns consistent with Wallerian degeneration of the axon, and terminal ovoids indicative of axonal transection (Figure C3-1). We detected a large infiltrate of macrophages/microglia in areas of demyelination, consistent with a role for these cells as the terminal effectors of MHV-induced demyelination. It seems likely that these cells also partly mediate axonal damage. These areas of demyelination had relatively little viral antigen staining, suggesting that myelin and axonal damage occurred during the process of viral clearance by infiltrating lymphocytes and macrophages/microglia.

Next we analyzed areas of white matter adjacent to demyelinating lesions. These periplaque regions exhibited abundant staining for virus antigen, with a modest infiltration of macrophages as compared to areas of demyelination. This most likely represents an early infiltrate of macrophages into virus-infected white matter. This infiltration of macrophages was accompanied by roughly half the level of staining for nonphosphoneurofilament $\mathrm{H}$ as detected in areas of frank demyelination.

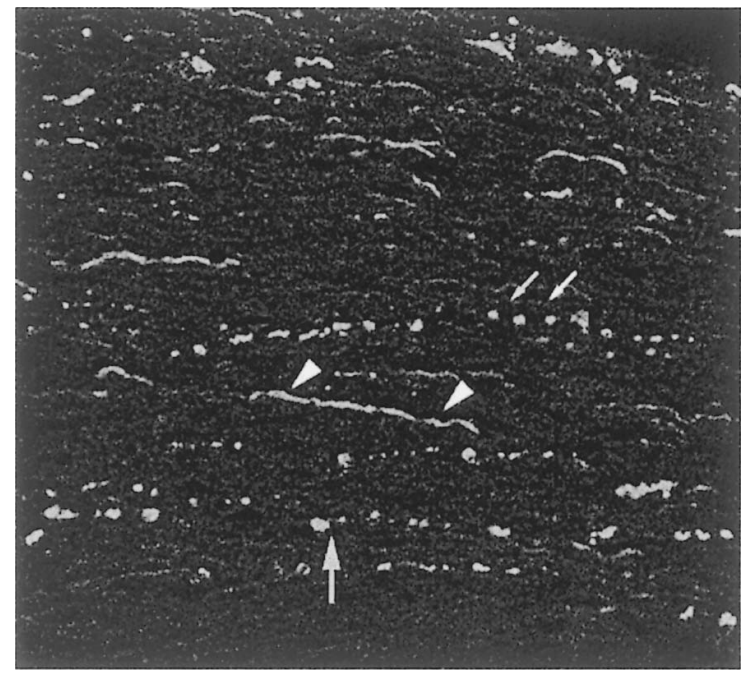

Figure C3-1. MHV-induced axonal damage. Midsagittal spinal cord sections from MHVinfected mice were stained with mAb SMI-32. An area of demyelination within the white matter is shown. Three different types of axonal pathology are indicated. Arrowheads: a demyelinated axon. Small arrows: a degenerating axon. Large arrow: a terminal ovoid, consistent with axonal transection. 
Areas of normal appearing white matter distant from demyelinating lesions, as assessed by staining with LFB, were generally devoid of macrophages and viral antigen, although scattered virus-infected cells were occasionally detected in these regions of the spinal cord. These areas had only limited staining with $\mathrm{mAb}$ SMI-32. This staining may have resulted from direct viral damage to the axon, since viral antigen has been detected throughout axons (19). Alternatively, and we believe more likely, axonal damage was occurring distal to a demyelinating lesion.

Two methods were used to quantify the amount of axonal damage in the spinal cords of MHV-infected mice. In one method, midsagittal sections of whole spinal cords were stained with mAb SMI-32, analyzed by confocal microscopy, photographed and digitalized. The numbers of pixels fluorescing above background were counted (Figure C3-2A). This method quantifies total damage throughout the spinal cord, but because the majority of the spinal cord does not exhibit demyelination, tended to blunt differences between samples. To address this issue, the number of SMI-32 positive blebs in areas of demyelination, areas adjacent to demyelination, or normal appearing white matter was counted in a blinded fashion (Figure C3-2B). Quantification of the amount of axonal damage (Figure C3-2B) revealed that there were roughly twice as many SMI-32 positive axons in areas of demyelination, on average, than in adjacent areas, while there was minimal damage in distant normal-appearing areas of white matter.

Finally, we investigated the kinetics of axonal damage in relationship to the appearance of demyelination. Spinal cords of mice at 4.5 days p.i. were
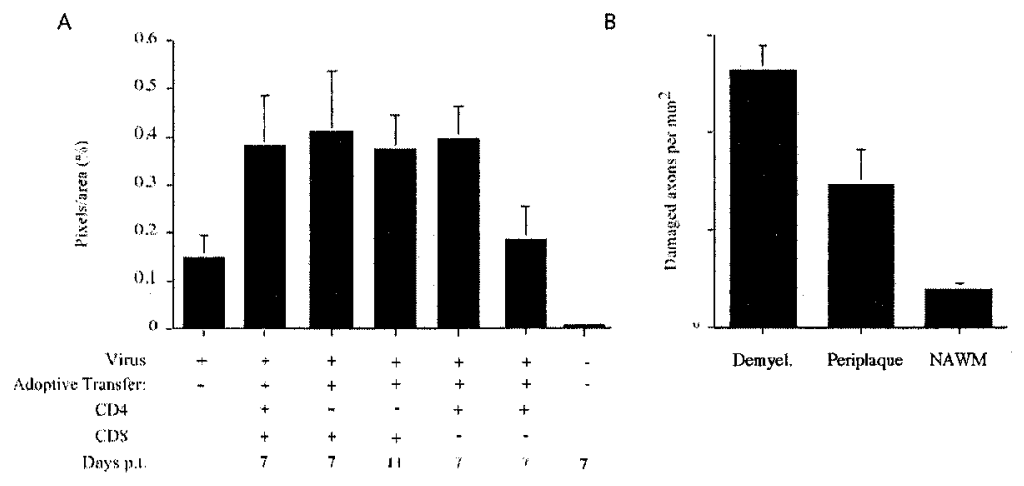

Figure C3-2. Quantification of axonal damage in MHV-infected mice. A, Whole spinal cords (midsagittal sections) were stained with SMI-32, photographed and digitalized, and the number of positive pixels was counted and expressed as a percentage of total spinal cord area. 
B, the number of terminal ovoids in areas of demyelination, areas adjacent to demyelination ("periplaque" areas) or areas of normal appearing white matter (NAWM) were counted.

analyzed using the stains described above. This is the first time point at which demyelination is detectable. Surprisingly, axonal damage, as measured by SMI-32 positivity, was found in these mice. This occurred only in areas of early macrophage infiltration and viral antigen, suggesting that demyelination and axonal damage occur concomitantly, and are mediated at least in part by the same effector agents.

\subsection{Contribution of CD4 and CD8 cells to axonal damage}

As previously published (16), there are substantial differences in clinical disease and in demyelination between recipients of CD4 T cell- and CD8 T cell-enriched splenic populations. We reasoned that these differences in phenotype could be related to differences in the amount of axonal damage mediated by these two cell types. Therefore, the adoptive transfer system was used to elucidate the contribution of $\mathrm{CD} 4$ and $\mathrm{CD} 8 \mathrm{~T}$ cells to the pathogenesis of axonal damage. MHV-infected $\mathrm{RAGl}^{-1-}$ mice received splenocytes enriched for either CD4 or CD8 T cells three days p.i. We quantified the amount of axonal damage by measuring the number of pixels above background in spinal cords stained with mAb SMI-32 and analyzed by confocal microscopy.

Mice receiving splenocytes depleted of both CD4 and CD8 T cells did not develop demyelination and also did not develop axonal damage (Figure C3-2A). Mice that received splenocytes treated with complement only exhibited $0.4 \%$ of mAb SMI-32 immunoreactivity across the spinal cord. Surprisingly, no difference in total mAb SMI-32 immunoreactivity could be detected between the spinal cords of recipients of CD4 T cell- or CD8 T cellenriched splenocytes. The amount of axonal damage was not different statistically from that observed in recipients of undepleted populations of cells. In addition, the amount of axonal damage was not well correlated with the extent of demyelination. These results show that axonal damage did not explain the differences in clinical disease observed between the recipients of undepleted and $\mathrm{T}$ cell subset-enriched splenocytes.

\section{FUTURE DIRECTIONS}

Axonal damage appears to be important in the pathological process in all demyelinating diseases, including $\mathrm{MS}, \mathrm{EAE}$, and viral models of 
demyelination, including MHV-infected mice (5, 10-13). In the model of MHV-induced demyelination described herein, clinical disease and demyelination occur rapidly and reproducibly after adoptive transfer of immune $\mathrm{T}$ cells, making it ideal for the dissection of the processes leading to axonal damage in demyelinating disease.

Although CD4 and CD8 T cells both contribute to demyelination and axonal damage, the mechanisms by which these processes are mediated remains to be determined. CD8 T cell-mediated demyelination is interferon- $\gamma$ mediated, whereas CD4 T cell-mediated demyelination is increased in the absence of this cytokine $(20,21)$. Demyelination itself is associated with changes in the cytoskeletal structure of the associated axon (22). In mice with genetic disruption of the proteolipid protein gene, axonal dysfunction is observed in the presence of normally compacted myelin $(23,24)$. In some models, demyelinated axons appear to be able to compensate for the loss of myelin by upregulating ion channels necessary to maintain conduction ( 25 , 26). Other demyelinated axons fail to do so, leading to severe conduction defects and axonal dysfunction. It is likely that $T$ cells and macrophages/microglia, by creating a pro-inflammatory milieu, contribute to the pathogenesis of axonal injury.

Important issues to be resolved include identifying the effector molecules and cells that cause axonal damage and determining if axonal injury is a necessary consequence of demyelination. Once the mechanisms of axonal dysfunction and damage are better understood, it may be possible to design therapy to minimize axonal disease in MHV-infected mice, and ultimately, in humans with MS.

\section{REFERENCES}

1. Noseworthy JH. Progress in determining the causes and treatment of multiple sclerosis. Nature. 1999;399:A40-A47.

2. Hemmer B, Archelos JJ, Hartung HP. New concepts in the immunopathogenesis of multiple sclerosis. Nat Rev Neurosci. 2002;3:291301.

3. Lassmann $\mathbf{H}$. The pathology of multiple sclerosis and its evolution. Philos Trans R Soc Lond B Biol Sci. 1999;354:1635-40.

4. De Stefano N, Matthews PM, Narayanan S, Francis GS, Antel JP, Arnold DL. Axonal dysfunction and disability in a relapse of multiple sclerosis: longitudinal study of a patient. Neurology. 1997;49:1138-41.

5. Trapp B, Peterson J, Ransohoff R, Rudick R, Monk S, Bo L. Axonal transection in the lesions of multiple sclerosis. New Engl. J. Med. 1998;338:278-285.

6. Ferguson B, Matyszak M, Esiri M, Perry V. Axonal damage in acute multiple sclerosis lesions. Brain. 1997;120:393-399. 
7. Matthews PM, De Stefano N, Narayanan S, et al. Putting magnetic resonance spectroscopy studies in context: axonal damage and disability in multiple sclerosis. Sem. Neurol. 1998;18:327-36.

8. Brex PA, Ciccarelli O, O'Riordan JI, Sailer M, Thompson AJ, Miller DH. A longitudinal study of abnormalities on MRI and disability from multiple sclerosis. $N$ Engl $J$ Med. 2002;346:158-64.

9. Waxman SG. Demyelinating diseases--new pathological insights, new therapeutic targets, $N$ Engl J Med. 1998;338:323-5.

10. Kornek B, Storch MK, Weissert R, et al. Multiple sclerosis and chronic autoimmune encephalomyelitis: a comparative quantitative study of axonal injury in active, inactive, and remyelinated lesions. Am J Pathol. 2000;157(1):267-76.

11. Raine CS, Cross AH. Axonal dystrophy as a consequence of longterm demyelination. Lab. Invest. 1989;60:714-725.

12. Sathornsumetee $\mathrm{S}$, McGavern DB, Ure DR, Rodriguez $M$. Quantitative ultrastructural analysis of a single spinal cord demyelinated lesion predicts total lesion load, axonal loss, and neurological dysfunction in a murine model of multiple sclerosis. Am J Pathol. 2000;157(4):136576.

13. Dandekar AA, Wu G, Pewe LL, Perlman S. Axonal damage is T cell mediated and occurs concomitantly with demyelination in mice infected with a neurotropic cornavirus. J. Virol. 2001;75:6115-6120.

14. Stohlman SA, Bergmann CC, Perlman S. Mouse hepatitis virus. In: Ahmed R, Chen I, eds. Persistent Viral Infections. New York: John Wiley \& Sons, Ltd.; 1998:537-557.

15. Haring J, Perlman S. Mouse hepatitis virus. Curr Opin Microbiol. 2001;4:462-6.

16. Wu GF, Dandekar AA, Pewe L, Perlman S. CD4 and CD8 T cells have redundant but not identical roles in virus-induced demyelination. $J$. Immunol. 2000;165:2278-2286.

17. Mombaerts P, Iacomini J, Johnson RS, Herrup K, Tonegawa S, Papaloannou VE. RAG-1-deficient mice have no mature $B$ and $T$ lymphocytes. Cell. 1992;68:869-877.

18. Xue S, Sun N, van Rooijen N, Perlman S. Depletion of blood-borne macrophages does not reduce demyelination in mice infected with a neurotropic coronavirus. J. Virol. 1999;73:6327-6334.

19. Pasick J, Kalicharran K, Dales S. Distribution and trafficking of JHM coronavirus structural proteins and virions in primary neurons and the OBL-21 neuronal cell line. J. Virol. 1994;68:2915-2928.

20. Pewe L, Haring J, Perlman S. CD4 T-cell-mediated demyelination is increased in the absence of gamma interferon in mice infected with mouse hepatitis virus. $J$ Virol. 2002;76:7329-33.

21. Pewe LL, Perlman S. CD8 T cell-mediated demyelination is IFN- $\gamma$ dependent in mice infected with a neurotropic coronavirus. $J$. Immunol. 2002;168:1547-1551.

22. Kirkpatrick LL, Brady ST. Modulation of the axonal microtubule cytoskeleton by myelinating Schwann cells. J Neurosci. 1994;14:7440-50.

23. Boison D, Stoffel W. Disruption of the compacted myelin sheath of axons of the central nervous system in proteolipid protein-deficient mice. Proc Natl Acad Sci U S A. 1994;91:11709-13. 
24. Griffiths I, Klugmann M, Anderson T, et al. Axonal swellings and degeneration in mice lacking the major proteolipid of myelin. Science. 1998;280:1610-1613.

25. Rivera-Quinones C, McGavern D, Schmelzer J, Hunter S, Low P, Rodriguez M. Absence of neurological deficits following extensive demyelination in a class I-deficient murine model of multiple sclerosis. Nature Med. 1998;4:187-193.

26. Felts PA, Baker TA, Smith KJ. Conduction in segmentally demyelinated mammalian central axons. J Neurosci. 1997;17:7267-77. 